\title{
Screening for antidepressant, sedative and analgesic activities of novel fused thiophene derivatives
}

\author{
WAGNAT W. WARDAKHAN ${ }^{1 *}$ \\ OMAR M. E. ABDEL-SALAM ${ }^{2}$ \\ GAMAL A. ELMEGEED ${ }^{2}$ \\ ${ }^{1}$ National Organization for \\ Drug Control and Research \\ P.O. 29 Cairo, Egypt \\ 2 Pharmacology Department \\ Hormone Department, National \\ Research Centre, Dokki, Cairo, Egypt
}

Accepted February 1, 2008

\begin{abstract}
This study was aimed at the synthesis of fused benzothiophene derivatives containing heterocyclic moiety. The reaction of the tetrahydrobenzo[b]thiophene derivatives $\mathbf{1} \mathbf{a}, \mathbf{b}$ with ethoxycarbonylisothiocyanate afforded the thiourea derivatives $\mathbf{2} \mathbf{a}, \mathbf{b}$. Cyclization of the latter products gave the annulated benzo[b]thienopyrimidine derivatives $\mathbf{3} \mathbf{a}, \mathbf{b}$. Compounds $\mathbf{2} \mathbf{a}, \mathbf{b}$ and $\mathbf{3} \mathbf{a}$ underwent $\mathbf{a}$ series of heterocyclization reactions through the reaction with some chemical reagents to give the new ben$\mathrm{zo}[\mathrm{b}]$ thienopyrimidine derivatives $\mathbf{5 a}, \mathbf{b}$ to $\mathbf{8 a}$-c. Also, this work was extended to study the potential role of the novel synthesized thiourea derivative $2 \mathbf{a}$ and benzo[b]thienopyrimidine derivatives $\mathbf{3 a}, \mathbf{5 b}, \mathbf{6} \mathbf{a}$ and $\mathbf{8 b}$ as antidepressant, sedative or analgesic agents at two doses (15 or 30 $\mathrm{mg} \mathrm{kg}{ }^{-1}$ body mass). Some compounds (2a, $3 \mathbf{a}$ and $5 \mathbf{b}$ ) showed mild antidepressant activity in the forced-swimming test. No compound showed sedative effect. Visceral pain evoked by i.p. injection of acetic acid in mice was significantly inhibited by all compounds at a high doses.
\end{abstract}

Keywords: tetrahydrobenzo[b]thiophene, pyrimidine, thiourea, antidepressant, sedative, analgesic

Heterocyclic antidepressants were the mainstay of antidepressant treatment from their inception in the late 1950's until the mid-1980's. The development of synthetic heterocyclic compounds as antidepressant, sedative or analgesic agents progressed considerably during the past decade (1-3). In recent years, the role of antidepressant drugs in the management of different pain syndromes has elicited considerable interest $(4,5)$. Pyrimidine derivatives possessing anti-inflammatory, antidepressant and analgesic activities have been reported in literature (6-8). Over the past few years, our research group was conducting a comprehensive program towards the synthesis of thiophenes and their fused derivatives (9-12). The importance of such compounds is based on their use as anti-flammatory $(13,14)$, anti-protozoal $(15)$, and antitumor agents $(16)$, templates for

\footnotetext{
* Correspondence, e-mail: wagnatward@hotmail.com
} 
W. W. Wardakhan et al:: Screening for antidepressant, sedative and analgesic activities of novel fused thiophene derivatives, Acta Pharm. 58 (2008) 1-14.

serine protease inhibition (17) and alternate substrate inhibitors of cholesterol eastrase (18). In view of all these observations, we report here in on the use of 2-amino-4,5,6,7-tetrahydrobenzo[b]thiophene derivatives $(\mathbf{1} \mathbf{a}, \mathbf{b})(19)$ in the synthesis of novel benzo[b]thienopyrimidine derivatives. The systemic anti-depressant, sedative or analgesic activities of the newly synthesized compounds were also investigated.

\section{EXPERIMENTAL}

\section{Synthetic methods, analytical and spectral data}

Melting points were determined on an electrothermal apparatus (Büchi 535, Switzerland) in an open capillary tube and are uncorrected. Elemental analyses were determined on a Yanaco CHN Corder elemental analyzer (Japan). IR spectra $\left(v \mathrm{~cm}^{-1}\right)$ were recorded in $\mathrm{KBr}$ pellets on a PA-9721 IR spectrophotometer (Shimadzu, Japan). ${ }^{1} \mathrm{H}$ NMR and ${ }^{13} \mathrm{C}$ NMR spectra were obtained on a Jeol $300 \mathrm{MHz}$ (Japan) spectrometer in DMSO$-\mathrm{d}_{6}$ as solvent, using TMS as internal reference and chemical shifts $(\delta)$ are expressed in ppm. Mass spectra were recorded on Kratos (75e V) MS equipment (Germany). Elemental analyzes were performed on a Yanaco CHN Corder elemental analyzes (Japan).

The analytical and spectral data of the newly synthesized products are presented in Tables I and II, respectively. The pharmacological data are given in Tables III, IV and V.

Compounds $\mathbf{2} \mathbf{a}$ and $\mathbf{2} \mathbf{b}$ were synthesized according to our recently published work (20).

3-Cyano-2-(N-ethoxycarbonylthiouryl)-4,5,6,7-tetrahydrobenzo[b]thiophene (2a) and ethyl 2-(N-ethoxycarbonylthiouryl)-4,5,6,7-tetrahydrobenzo[b]thiophen-3-carboxylate $(2 \boldsymbol{b})$

General procedure. - To a solution of either $1 \mathrm{a}(1.78 \mathrm{~g}, 0.01 \mathrm{~mol})$ or $\mathbf{1 b}(2.25 \mathrm{~g}, 0.01$ $\mathrm{mol})$ in 1,4-dioxan $(30 \mathrm{~mL})$, ethoxycarbonyl isothiocyanate $(1.31 \mathrm{~g}, 0.01 \mathrm{~mol})$ [prepared by adding ammonium isothiocyanate $(0.01 \mathrm{~mol})$ to a solution of ethyl chloroformate $(0.01 \mathrm{~mol})$ in 1,4-dioxan $(20 \mathrm{~mL})$ and heating for $1 / 2 \mathrm{~h}$ followed by isolation of the byproduct, ammonium chloride] was added. The whole reaction mixture, in each case, was stirred at room temperature overnight and the solid product formed upon pouring onto ice/water was collected by filtration to give either $\mathbf{2} \mathbf{a}$ or $\mathbf{2} \mathbf{b}$, crystallized from acetic acid.

Ethyl 1-imino-3-thioxo-4[H]-6,7,8,9-tetrahydrobenzo[b]thieno[2,3-d]pyrimidin-2-carboxylate (3a) and ethyl 1-oxo-3-thioxo-4[H]-6,7,8,9-tetrahydrobenzo[b]thieno[2,3-d]pyrimidin-2-carboxylate (3b)

General procedure. - A suspension of either $2 \mathbf{a}(3.09 \mathrm{~g}, 0.01 \mathrm{~mol})$ or $2 \mathbf{b}(3.56 \mathrm{~g}, 0.01$ $\mathrm{mol})$ in sodium ethoxide $(0.01 \mathrm{~mol})$ [prepared by dissolving sodium metal $(0.23 \mathrm{~g}, 0.01$ $\mathrm{mol})$ in absolute ethanol $(40 \mathrm{~mL})$ ] was heated in a boiling water bath for $6 \mathrm{~h}$ and then left to cool. The solid product, formed upon pouring onto ice/water containing hydrochloric acid (to $\mathrm{pH}=6$ ), was collected by filtration to give either $3 a$, crystallized from 1,4-dioxane or $3 b$, crystallized from ethanol. 
W. W. Wardakhan et al:: Screening for antidepressant, sedative and analgesic activities of novel fused thiophene derivatives, Acta Pharm. 58 (2008) 1-14.

3-Cyano-2-(N-phenylaminocarbonylthiourayl-4,5,6,7-tetrahydrobenzo[b]thiophene (4a) and ethyl2-(N-phenylaminocarbonylthiourayl-4,5,6,7-tetrahydrobenzo[b]thiophen-3-carboxylate (4b)

General procedure. - To a dry solid of either 2a (3.09 g, $0.01 \mathrm{~mol})$ or $2 \mathbf{b}(3.56 \mathrm{~g}, 0.01$ $\mathrm{mol})$, aniline oil $(0.94 \mathrm{~g}, 0.01 \mathrm{~mol})$ was added. The reaction mixture was heated in an oil

Table I. Physico-chemical data for the synthesized compounds

\begin{tabular}{|c|c|c|c|c|c|c|c|}
\hline \multirow{2}{*}{$\begin{array}{c}\text { Compd. } \\
\text { No. }\end{array}$} & \multirow{2}{*}{$\begin{array}{l}\text { Yield } \\
(\%)\end{array}$} & \multirow{2}{*}{$\begin{array}{l}\text { M.p. } \\
\left({ }^{\circ} \mathrm{C}\right)\end{array}$} & \multirow{2}{*}{$\begin{array}{l}\text { Mol. formula } \\
\qquad\left(M_{\mathrm{r}}\right)\end{array}$} & \multicolumn{4}{|c|}{ Found/calcd. (\%) } \\
\hline & & & & $\mathrm{C}$ & $\mathrm{H}$ & $\mathrm{N}$ & $S$ \\
\hline \multirow[t]{2}{*}{$2 a$} & \multirow[t]{2}{*}{70} & \multirow[t]{2}{*}{$188-190$} & \multirow{2}{*}{$\begin{array}{c}\mathrm{C}_{13} \mathrm{H}_{15} \mathrm{~N}_{3} \mathrm{O}_{2} \mathrm{~S}_{3} \\
(309.41)\end{array}$} & 50.46 & 4.89 & 13.58 & 20.73 \\
\hline & & & & 50.07 & 5.42 & 13.88 & 20.57 \\
\hline \multirow[t]{2}{*}{$2 b$} & \multirow[t]{2}{*}{66} & \multirow[t]{2}{*}{105} & \multirow{2}{*}{$\begin{array}{c}\mathrm{C}_{15} \mathrm{H}_{20} \mathrm{~N}_{2} \mathrm{O}_{4} \mathrm{~S}_{2} \\
(356.46)\end{array}$} & 50.54 & 5.66 & 7.86 & 17.99 \\
\hline & & & & 50.87 & 5.24 & 8.31 & 18.44 \\
\hline \multirow[t]{2}{*}{$3 a$} & \multirow[t]{2}{*}{62} & \multirow[t]{2}{*}{$233-235$} & \multirow{2}{*}{$\begin{array}{c}\mathrm{C}_{13} \mathrm{H}_{15} \mathrm{~N}_{3} \mathrm{O}_{2} \mathrm{~S}_{2} \\
(309.41)\end{array}$} & 50.46 & 4.89 & 13.58 & 20.73 \\
\hline & & & & 50.22 & 5.31 & 13.88 & 21.12 \\
\hline \multirow[t]{2}{*}{$3 b$} & \multirow[t]{2}{*}{55} & \multirow[t]{2}{*}{$233-235$} & \multirow{2}{*}{$\begin{array}{c}\mathrm{C}_{13} \mathrm{H}_{14} \mathrm{~N}_{2} \mathrm{O}_{3} \mathrm{~S}_{2} \\
(310.40)\end{array}$} & 50.30 & 4.54 & 9.02 & 20.66 \\
\hline & & & & 50.07 & 4.88 & 20.38 & 20.38 \\
\hline \multirow[t]{2}{*}{$4 a$} & \multirow[t]{2}{*}{84} & \multirow[t]{2}{*}{$158-162$} & \multirow{2}{*}{$\begin{array}{c}\mathrm{C}_{17} \mathrm{H}_{16} \mathrm{~N}_{4} \mathrm{OS}_{2} \\
(356.47)\end{array}$} & 57.28 & 4.52 & 15.72 & 17.99 \\
\hline & & & & 56.89 & 4.33 & 15.92 & 18.31 \\
\hline \multirow[t]{2}{*}{$4 b$} & \multirow[t]{2}{*}{73} & \multirow[t]{2}{*}{$229-233$} & $\mathrm{C}_{19} \mathrm{H}_{21} \mathrm{~N}_{3} \mathrm{O}_{3} \mathrm{~S}_{2}$ & 56.55 & 5.25 & 10.41 & 15.89 \\
\hline & & & $(403.53)$ & 56.39 & 5.09 & 10.22 & 15.89 \\
\hline $5 a$ & 61 & $>300$ & $\mathrm{C}_{17} \mathrm{H}_{16} \mathrm{~N}_{4} \mathrm{OS}_{2}$ & 57.28 & 4.52 & 15.72 & 17.99 \\
\hline & & & (356.47) & 56.79 & 4.42 & 16.02 & 18.33 \\
\hline $5 b$ & 52 & $266-270$ & $\mathrm{C}_{17} \mathrm{H}_{15} \mathrm{~N}_{3} \mathrm{O}_{2} \mathrm{~S}_{2}$ & 57.12 & 4.23 & 11.76 & 17.94 \\
\hline & & & $(357.46)$ & 56.79 & 4.09 & 11.46 & 18.31 \\
\hline $6 a$ & 74 & 198-200 & $\mathrm{C}_{25} \mathrm{H}_{22} \mathrm{~N}_{4} \mathrm{O}_{2} \mathrm{~S}_{2}$ & 63.27 & 4.67 & 11.80 & 13.51 \\
\hline & & & $(474.61)$ & 62.99 & 4.82 & 11.67 & 13.72 \\
\hline $6 b$ & 62 & $145-147$ & $\mathrm{C}_{25} \mathrm{H}_{21} \mathrm{~N}_{3} \mathrm{O}_{3} \mathrm{~S}_{2}$ & 63.14 & 4.45 & 8.84 & 13.48 \\
\hline & & & $(475.59)$ & 62.89 & 4.75 & 9.24 & 13.69 \\
\hline $7 a$ & 56 & $222-226$ & $\mathrm{C}_{21} \mathrm{H}_{22} \mathrm{~N}_{4} \mathrm{O}_{3} \mathrm{~S}_{2}$ & 56.99 & 5.01 & 12.66 & 14.49 \\
\hline & & & $(442.56)$ & 56.89 & 4.76 & 12.93 & 14.83 \\
\hline $7 \mathrm{~b}$ & 75 & 189-191 & $\mathrm{C}_{21} \mathrm{H}_{21} \mathrm{~N}_{3} \mathrm{O}_{4} \mathrm{~S}_{2}$ & 56.87 & 4.77 & 9.47 & 14.46 \\
\hline & & & $(443.55)$ & 56.67 & 5.08 & 9.34 & 14.33 \\
\hline $8 a$ & 52 & $136-138$ & $\mathrm{C}_{17} \mathrm{H}_{18} \mathrm{~N}_{6} \mathrm{OS}$ & 57.61 & 5.12 & 23.71 & 9.05 \\
\hline & & & $(354.44)$ & 57.33 & 5.63 & 23.59 & 8.88 \\
\hline $8 b$ & 63 & $220-223$ & $\mathrm{C}_{23} \mathrm{H}_{22} \mathrm{~N}_{6} \mathrm{OS}$ & 64.16 & 5.15 & 9.52 & 17.45 \\
\hline & & & $(430.53)$ & 63.77 & 4.98 & 19.78 & 7.72 \\
\hline $8 c$ & 70 & $170-172$ & $\mathrm{C}_{17} \mathrm{H}_{17} \mathrm{~N}_{5} \mathrm{O}_{2} \mathrm{~S}$ & 57.44 & 4.82 & 19.70 & 9.02 \\
\hline & & & $(355.42)$ & 57.28 & 4.59 & 19.58 & 8.81 \\
\hline $8 d$ & 50 & $>300$ & $\mathrm{C}_{23} \mathrm{H}_{21} \mathrm{~N}_{5} \mathrm{O}_{2} \mathrm{~S}$ & 64.02 & 4.90 & 16.23 & 7.43 \\
\hline & & & $(431.52)$ & 63.89 & 5.38 & 16.39 & 8.59 \\
\hline
\end{tabular}


W. W. Wardakhan et al.: Screening for antidepressant, sedative and analgesic activities of novel fused thiophene derivatives, Acta Pharm. 58 (2008) 1-14.

Table II. Spectral data of the synthesized products

\begin{tabular}{|c|c|c|c|c|}
\hline $\begin{array}{c}\text { Compd. } \\
\text { No. }\end{array}$ & $\begin{array}{l}\mathrm{IR} \\
\left(v, \mathrm{~cm}^{-1}\right)\end{array}$ & $\begin{array}{l}{ }^{1} \mathrm{H} \text { NMR } \\
(\delta, \mathrm{ppm})\end{array}$ & $\begin{array}{l}{ }^{13} \mathrm{C} \text { NMR } \\
(\delta, \mathrm{ppm})\end{array}$ & $\begin{array}{c}\text { MS } \\
\left(\mathrm{M}^{+}\right)\end{array}$ \\
\hline $2 a$ & $\begin{array}{l}3460-3324(2 \mathrm{NH}), \\
2980,2888\left(\mathrm{CH}_{3},\right. \\
\left.\mathrm{CH}_{2}\right), 2225(\mathrm{CN}), 1687 \\
(\mathrm{CO}), 1638(\mathrm{C}=\mathrm{C}) \\
1205-1196(\mathrm{C}=\mathrm{S})\end{array}$ & $\begin{array}{l}1.61(\mathrm{t}, 3 \mathrm{H}, J=7.02 \mathrm{~Hz}, \\
\left.\mathrm{CH}_{3}\right), 2.14-2.16(\mathrm{~m}, 4 \mathrm{H}, \\
\left.2 \mathrm{CH}_{2}\right), 2.23-2.26(\mathrm{~m}, 4 \mathrm{H}, \\
\left.2 \mathrm{CH}_{2}\right), 4.11(\mathrm{~s}, 1 \mathrm{H}, \mathrm{NH}) \\
4.24\left(\mathrm{q}, 2 \mathrm{H}, J=7.02, \mathrm{CH}_{2}\right) \\
8.32(\mathrm{~s}, 1 \mathrm{H}, \mathrm{NH})\end{array}$ & $\begin{array}{l}14.88\left(\text { ester } \mathrm{CH}_{3}\right), 20.0, \\
23.3,23.9,24.7\left(4 \mathrm{CH}_{2}\right), \\
60.5\left(\text { ester } \mathrm{CH}_{2}\right), 118.8 \\
(\mathrm{CN}), 122.3,136.7,135.6, \\
\text { 140.8 (thiophene-C), } 154.7 \\
\text { (amide } \mathrm{C}=\mathrm{O}), 178.8(\mathrm{C}=\mathrm{S})\end{array}$ & 309 \\
\hline $2 b$ & $\begin{array}{l}3456-3339(2 \mathrm{NH}), \\
2986,2893\left(\mathrm{CH}_{3},\right. \\
\left.\mathrm{CH}_{2}\right), 1690,1685(2 \\
\mathrm{CO}), 1636(\mathrm{C}=\mathrm{C}) \\
1205-1196(\mathrm{C}=\mathrm{S})\end{array}$ & $\begin{array}{l}1.62,1.65\left(2 \mathrm{t}, 6 \mathrm{H}, 2 \mathrm{CH}_{3}\right) \\
2.16-2.19\left(\mathrm{~m}, 4 \mathrm{H}, 2 \mathrm{CH}_{2}\right), \\
2.25-2.29\left(\mathrm{~m}, 4 \mathrm{H}, 2 \mathrm{CH}_{2}\right) \\
4.10(\mathrm{~s}, 1 \mathrm{H}, \mathrm{NH}), 4.22,4.25 \\
\left(2 \mathrm{q}, 4 \mathrm{H}, 2 \mathrm{CH}_{2}\right), 8.30(\mathrm{~s}, 1 \mathrm{H}, \\
\mathrm{NH})\end{array}$ & $\begin{array}{l}14.87,16.58 \text { (two ester } \\
\left.\mathrm{CH}_{3}\right), 20.1,23.5,23.6,24.4 \\
\left(4 \mathrm{CH}_{2}\right), 60.6,62.7 \text { (two ester } \\
\left.\mathrm{CH}_{2}\right), 118.6(\mathrm{CN}), 122.1 \text {, } \\
136.3,135.9,140.1 \text { (thiop- } \\
\text { hene-C), } 154.6 \text { (amide } \\
\mathrm{C}=\mathrm{O}), 179.5(\mathrm{C}=\mathrm{S})\end{array}$ & 356 \\
\hline $3 a$ & $\begin{array}{l}3442-3326(2 \mathrm{NH}), \\
2982,2887\left(\mathrm{CH}_{3},\right. \\
\left.\mathrm{CH}_{2}\right), 1688(\mathrm{CO}), 1665 \\
(\text { exocyclic } \mathrm{C}=\mathrm{N}), 1639 \\
(\mathrm{C}=\mathrm{C}), 1207-1193 \\
(\mathrm{C}=\mathrm{S})\end{array}$ & $\begin{array}{l}1.36(\mathrm{t}, 3 \mathrm{H}, J=7.66 \mathrm{~Hz} \\
\left.\mathrm{CH}_{3}\right), 1.69-1.72(\mathrm{~m}, 4 \mathrm{H}, \\
\left.2 \mathrm{CH}_{2}\right), 2.20-2.23(\mathrm{~m}, 4 \mathrm{H}, \\
\left.2 \mathrm{CH}_{2}\right), 4.13(\mathrm{~s}, 1 \mathrm{H}, \mathrm{NH}) \\
4.20(\mathrm{q}, 2 \mathrm{H}, J=7.66 \mathrm{~Hz} \\
\left.\mathrm{CH}_{2}\right), 8.26(\mathrm{~s}, 1 \mathrm{H}, \mathrm{NH})\end{array}$ & $\begin{array}{l}13.8\left(\text { ester } \mathrm{CH}_{3}\right), 23.3,23.9, \\
24.7\left(4 \mathrm{CH}_{2}\right), 52.7 \\
\left(\text { ester } \mathrm{CH}_{2}\right), 121.3,134.3, \\
136.9,147.9 .1(\text { thiophene-C), } \\
159.8(\mathrm{C}=\mathrm{O}), 164.7(\mathrm{C}=\mathrm{N}) \text {, } \\
179.5(\mathrm{C}=\mathrm{S})\end{array}$ & 309 \\
\hline $3 b$ & $\begin{array}{l}3456-3336(\mathrm{NH}), 2980, \\
2890\left(\mathrm{CH}_{3}, \mathrm{CH}_{2}\right), \\
1693,1685(2 \mathrm{CO}), \\
1636(\mathrm{C}=\mathrm{C}), 1204-1190 \\
(\mathrm{C}=\mathrm{S})\end{array}$ & $\begin{array}{l}1.38(\mathrm{t}, 3 \mathrm{H}, J=7.21 \mathrm{~Hz} \\
\left.\mathrm{CH}_{3}\right), 1.66-1.70(\mathrm{~m}, 4 \mathrm{H}, \\
\left.2 \mathrm{CH}_{2}\right), 2.22-2.26(\mathrm{~m}, 4 \mathrm{H}, \\
\left.2 \mathrm{CH}_{2}\right), 4.22(\mathrm{~s}, 1 \mathrm{H}, \mathrm{NH}) \\
4.27(\mathrm{q}, 2 \mathrm{H}, J=7.21 \mathrm{~Hz} \\
\left.\mathrm{CH}_{2}\right)\end{array}$ & & 310 \\
\hline $4 a$ & $\begin{array}{l}3489-3326(3 \mathrm{NH}), \\
3053(\mathrm{CH} \text {-aromatic), } \\
2218(\mathrm{CN}), 1682(\mathrm{CO}), \\
1637(\mathrm{C}=\mathrm{C})\end{array}$ & $\begin{array}{l}1.64-1.68\left(\mathrm{~m}, 4 \mathrm{H}, 2 \mathrm{CH}_{2}\right) \\
2.25-2.28\left(\mathrm{~m}, 4 \mathrm{H}, 2 \mathrm{CH}_{2}\right) \\
4.33,5.42(2 \mathrm{~s}, 2 \mathrm{H}, 2 \mathrm{NH}) \\
7.03-7.35\left(\mathrm{~m}, 5 \mathrm{H}, \mathrm{C}_{6} \mathrm{H}_{5}\right) \\
8.81(\mathrm{~s}, 1 \mathrm{H}, \mathrm{NH})\end{array}$ & $\begin{array}{l}\text { 20.3, 22.3, 23.9, } 24.9 \\
\left(4 \mathrm{CH}_{2}\right), 118.6(\mathrm{CN}), 120.8, \\
121.7,122.8,124.7,137.9 \\
\text { (phenyl C), 130.7, 132.2, } \\
\text { 138.7, 144.8 (thiophene C), } \\
\text { 155.8 (CO), 178.3 (C=S) }\end{array}$ & 356 \\
\hline $4 b$ & $\begin{array}{l}3487-3320(3 \mathrm{NH}), \\
3056(\mathrm{CH} \text {-aromatic }), \\
2988,2873\left(\mathrm{CH}_{3 \prime}\right. \\
\left.\mathrm{CH}_{2}\right), 1692,1686(2 \\
\mathrm{CO}), 1637(\mathrm{C}=\mathrm{C})\end{array}$ & $\begin{array}{l}1.34(\mathrm{t}, 3 \mathrm{H}, J=6.77 \mathrm{~Hz}, \\
\left.\mathrm{CH}_{3}\right), 1.66-1.72(\mathrm{~m}, 4 \mathrm{H}, \\
\left.2 \mathrm{CH}_{2}\right), 2.23-2.27(\mathrm{~m}, 4 \mathrm{H}, \\
\left.2 \mathrm{CH}_{2}\right), 4.22(\mathrm{q}, 2 \mathrm{H}, J=6.77 \\
\left.\mathrm{Hz}, \mathrm{CH}_{2}\right), 4.30,5.30(2 \mathrm{~s}, 2 \mathrm{H}, \\
\left.2 \mathrm{NH}^{2}\right), 7.33-7.42(\mathrm{~m}, 5 \mathrm{H}, \\
\left.\mathrm{C}_{6} \mathrm{H}_{5}\right), 8.94(\mathrm{~s}, 1 \mathrm{H}, \mathrm{NH})\end{array}$ & & 403 \\
\hline $5 a$ & $\begin{array}{l}3462-3336(3 \mathrm{NH}), \\
3059(\mathrm{CH}-\text { aromatic }), \\
1681(\mathrm{CO}), 1670 \text { (exo- } \\
\text { cyclic } \mathrm{C}=\mathrm{N}), 1644 \\
(\mathrm{C}=\mathrm{C}), 1205-1192 \\
(\mathrm{C}=\mathrm{S})\end{array}$ & $\begin{array}{l}1.68-1.70\left(\mathrm{~m}, 4 \mathrm{H}, 2 \mathrm{CH}_{2}\right), \\
2.25-2.29\left(\mathrm{~m}, 4 \mathrm{H}, 2 \mathrm{CH}_{2}\right), \\
4.32,5.36(2 \mathrm{~s}, 2 \mathrm{H}, 2 \mathrm{NH}), \\
7.22-7.36\left(\mathrm{~m}, 5 \mathrm{H}, \mathrm{C}_{6} \mathrm{H}_{5}\right), \\
9.05(\mathrm{~s}, 1 \mathrm{H}, \mathrm{NH})\end{array}$ & & 356 \\
\hline
\end{tabular}


W. W. Wardakhan et al.: Screening for antidepressant, sedative and analgesic activities of novel fused thiophene derivatives, Acta Pharm. 58 (2008) 1-14

$5 \mathbf{b}$

3462-3336 (2 NH), 3049 (CH-aromatic), 1693, 1685 (2 CO), $1637(\mathrm{C}=\mathrm{C}), 1203-1196$ $(\mathrm{C}=\mathrm{S})$

$6 a$

$3455-3330(2 \mathrm{NH})$, 3064 (CH-aromatic), 1690, 1687 (2CO) 1677 (exocyclic $\mathrm{C}=\mathrm{N})$, $1639(\mathrm{C}=\mathrm{C})$

(n)

$1.65-1.74\left(\mathrm{~m}, 4 \mathrm{H}, 2 \mathrm{CH}_{2}\right)$,

$2.22-2.27\left(\mathrm{~m}, 4 \mathrm{H}, 2 \mathrm{CH}_{2}\right)$, $4.36(\mathrm{~s}, 1 \mathrm{H}, \mathrm{NH}), 7.28-7.38$ $\left(\mathrm{m}, 5 \mathrm{H}, \mathrm{C}_{6} \mathrm{H}_{5}\right), 9.17(\mathrm{~s}, 1 \mathrm{H}$, $\mathrm{NH})$

$1.65-1.70\left(\mathrm{~m}, 4 \mathrm{H}, 2 \mathrm{CH}_{2}\right)$, $2.22-2.31\left(\mathrm{~m}, 4 \mathrm{H}, 2 \mathrm{CH}_{2}\right)$, $4.68\left(\mathrm{~s}, 2 \mathrm{H}, \mathrm{CH}_{2}\right), 7.33-7.39$ $\left(\mathrm{m}, 10 \mathrm{H}, 2 \mathrm{C}_{6} \mathrm{H}_{5}\right), 8.38,8.94$ $(2 \mathrm{~s}, 2 \mathrm{H}, 2 \mathrm{NH})$

6b $\quad 3442-3321(\mathrm{NH}), 3052 \quad 1.66-1.73\left(\mathrm{~m}, 4 \mathrm{H}, 2 \mathrm{CH}_{2}\right)$, (CH-aromatic), $\quad 2.24-2.28\left(\mathrm{~m}, 4 \mathrm{H}, 2 \mathrm{CH}_{2}\right)$, 1693-1685 (3CO), 16364.58 (s, 2H, $\left.\mathrm{CH}_{2}\right), 7.31-7.40$ $(\mathrm{C}=\mathrm{C})$ $\left(\mathrm{m}, 10 \mathrm{H}, 2 \mathrm{C}_{6} \mathrm{H}_{5}\right), 8.33(\mathrm{~s}$, $1 \mathrm{H}, \mathrm{NH})$

7a $\quad 3456-3318(2 \mathrm{NH})$, 3058 (CH-aromatic), 2978, $2869\left(\mathrm{CH}_{3}\right.$, $\left.\mathrm{CH}_{2}\right), 1690,1688$ (2 CO), $1660(\mathrm{C}=\mathrm{N})$, $1636(\mathrm{C}=\mathrm{C})$

$1.33(\mathrm{t}, 3 \mathrm{H}, J=7.11 \mathrm{~Hz}$ $\left.\mathrm{CH}_{3}\right), 1.64-1.71(\mathrm{~m}, 4 \mathrm{H}$, $\left.2 \mathrm{CH}_{2}\right), 2.25-2.32(\mathrm{~m}, 4 \mathrm{H}$, $\left.2 \mathrm{CH}_{2}\right), 4.21(\mathrm{q}, 2 \mathrm{H}, \mathrm{J}=7.11$ $\left.\mathrm{Hz}, \mathrm{CH}_{2}\right), 4.92\left(\mathrm{~s}, 2 \mathrm{H}, \mathrm{CH}_{2}\right)$, 7.26-7.37 (m, 5H, $\left.\mathrm{C}_{6} \mathrm{H}_{5}\right)$, $8.26,8.36(2 \mathrm{~s}, 2 \mathrm{H}, 2 \mathrm{NH})$

$7 \mathrm{~b}$

3447-3322 (NH), $30601.31(\mathrm{t}, 3 \mathrm{H}, J=7.04 \mathrm{~Hz}$, (CH-aromatic), 2982, $\left.\mathrm{CH}_{3}\right), 1.65-1.73(\mathrm{~m}, 4 \mathrm{H}$, $\left.2871\left(\mathrm{CH}_{3}, \mathrm{CH}_{2}\right), \quad 2 \mathrm{CH}_{2}\right), 2.24-2.36(\mathrm{~m}, 4 \mathrm{H}$, $1691,1688-1685\left(3 \quad 2 \mathrm{CH}_{2}\right), 4.24(\mathrm{q}, 2 \mathrm{H}, \mathrm{J}=7.04$ $\mathrm{CO}), 1656(\mathrm{C}=\mathrm{N})$, $1634(\mathrm{C}=\mathrm{C})$

$\left.\mathrm{Hz}, \mathrm{CH}_{2}\right), 4.86\left(\mathrm{~s}, 2 \mathrm{H}, \mathrm{CH}_{2}\right)$, 7.28-7.40 (m, 5H, $\left.\mathrm{C}_{6} \mathrm{H}_{5}\right)$, $8.33(\mathrm{~s}, 1 \mathrm{H}, \mathrm{NH})$

8a $3521-3312(3 \mathrm{NH}$, $\left.\mathrm{NH}_{2}\right), 3056$ (CH-aromatic), 1683 (CO), $1.65-1.70\left(\mathrm{~m}, 4 \mathrm{H}, 2 \mathrm{CH}_{2}\right)$, 2.22-2.31 (m, $\left.4 \mathrm{H}, 2 \mathrm{CH}_{2}\right)$, $2.88(\mathrm{~s}, 1 \mathrm{H}, \mathrm{NH}), 4.46(\mathrm{~s}$, 1670 (exocyclic $\left.\mathrm{C}=\mathrm{N}), \quad 2 \mathrm{H}, \mathrm{NH}_{2}\right), 6.58(\mathrm{~s}, 1 \mathrm{H}, \mathrm{NH})$, $1637(\mathrm{C}=\mathrm{C})$

7.31-7.39 (m, 5H, $\left.\mathrm{C}_{6} \mathrm{H}_{5}\right)$, $8.21(\mathrm{~s}, 1 \mathrm{H}, \mathrm{NH})$

$1.63-1.68\left(\mathrm{~m}, 4 \mathrm{H}, 2 \mathrm{CH}_{2}\right)$, $\begin{array}{ll}\left.\mathrm{NH}_{2}\right), 3062(\mathrm{CH}-a r o- & 2.24-2.36\left(\mathrm{~m}, 4 \mathrm{H}, 2 \mathrm{CH}_{2}\right), \\ \text { matic), } 1687(\mathrm{CO}), & 2.87,(\mathrm{~s}, 1 \mathrm{H}, 1 \mathrm{NH}), 6.55( \\ 1668 \text { (exocyclic } \mathrm{C}=\mathrm{N}), & 1 \mathrm{H}, \mathrm{NH}), 7.26-7.38(\mathrm{~m},\end{array}$ $\begin{array}{ll}\left.\mathrm{NH}_{2}\right), 3062(\mathrm{CH}-a \text { aro- } & 2.24-2.36\left(\mathrm{~m}, 4 \mathrm{H}, 2 \mathrm{CH}_{2}\right), \\ \text { matic), } 1687(\mathrm{CO}), & 2.87,(\mathrm{~s}, 1 \mathrm{H}, 1 \mathrm{NH}), 6.55(\mathrm{~s}, \\ 1668 \text { (exocyclic } \mathrm{C}=\mathrm{N}), & 1 \mathrm{H}, \mathrm{NH}), 7.26-7.38(\mathrm{~m},\end{array}$ 1668 (exocyclic $\mathrm{C}=\mathrm{N}), 1 \mathrm{H}, \mathrm{NH}), 7.26-7.38(\mathrm{~m}$, $1636(\mathrm{C}=\mathrm{C})$ $10 \mathrm{H}, 2 \mathrm{C}_{6} \mathrm{H}_{5}$ ), 8.20, 8.23 (2s, $2 \mathrm{H}, 2 \mathrm{NH})$

$8 c \quad 3560-3335(2 \mathrm{NH}$, $\left.\mathrm{NH}_{2}\right), 3052(\mathrm{CH}$-aromatic), 1691, 1688 (2 $\mathrm{CO}), 1657(\mathrm{C}=\mathrm{N})$, $1644(\mathrm{C}=\mathrm{C})$
1.60-1.66 (m, 4H, 2CH $)$, $2.20-2.32\left(\mathrm{~m}, 4 \mathrm{H}, 2 \mathrm{CH}_{2}\right)$, $2.87(\mathrm{~s}, 1 \mathrm{H}, \mathrm{NH}), 4.68(\mathrm{~s}$, $\left.2 \mathrm{H}, \mathrm{NH}_{2}\right), 7.32-7.37(\mathrm{~m}, 5 \mathrm{H}$, $\left.\mathrm{C}_{6} \mathrm{H}_{5}\right), 8.20(\mathrm{~s}, 1 \mathrm{H}, \mathrm{NH})$
$20.4,22.3,24.4,24.5$

$\left(4 \mathrm{CH}_{2}\right), 128.6(\mathrm{CN}), 120.6$,

$121.9,122.5,124.2,138.4$,

139.5, 140.3 (two phenyl C), $130.4,132.8,134.8$, 149.8 (thiophene C), 156.8 160.3 (2 CO), $168.7(\mathrm{C}=\mathrm{O})$

$20.9,22.6,24.4,24.9$

$\left(4 \mathrm{CH}_{2}\right), 120.8,121.7,122.8$, 123.5, 134.8 (phenyl C), 133.5, 133.7, 134.4, 147.9, 158.4 (thiophene, pyrimidine $\mathrm{C}), 158.9$ (2 CO), $169.3(\mathrm{C}=\mathrm{N})$ 
W. W. Wardakhan et al.: Screening for antidepressant, sedative and analgesic activities of novel fused thiophene derivatives, Acta Pharm. 58 (2008) 1-14.

$\begin{array}{lll}8 d & 1.62-1.69\left(\mathrm{~m}, 4 \mathrm{H}, 2 \mathrm{CH}_{2}\right), \\ 3348-3320(3 \mathrm{NH}), & 2.22-2.30\left(\mathrm{~m}, 4 \mathrm{H}, 2 \mathrm{CH}_{2}\right), \\ & 2.90(\mathrm{~s}, 1 \mathrm{H}, \mathrm{NH}), 7.31-7.42 \\ 1684,1680(2 \mathrm{CO}), & \left(\mathrm{m}, 10 \mathrm{H}, 2 \mathrm{C}_{6} \mathrm{H}_{5}\right), 8.22,8.24 \\ 1662(\mathrm{C}=\mathrm{N}), 1637 & (2 \mathrm{~s}, 2 \mathrm{H}, 2 \mathrm{NH}) \\ (\mathrm{C}=\mathrm{C}) & \end{array}$

bath $\left(140^{\circ} \mathrm{C}\right)$ for $1 \mathrm{~h}$. Then, it was left to cool and the remaining product was triturated with diethyl ether and the formed solid product was collected by filtration to give either $4 \mathbf{a}$ or $\mathbf{4 b}$, crystallized from acetic acid.

1-Imino-2-phenylaminocarbonyl-4[H]-3-thioxo-6,7,8,9-tetrahydrobenzo[b]thieno[2,3-d]pyrimidine $(5 a)$ and 1-oxo-2-phenylaminocarbonyl-4[H]-3-thioxo-6,7,8,9-tetrahydrobenzo[b]thieno$-[2,3-d]$ pyrimidine $(5 \boldsymbol{b})$

Method A. - A suspension of either $3 \mathrm{a}(3.56 \mathrm{~g}, 0.01 \mathrm{~mol})$ or $3 \mathrm{~b}(4.03 \mathrm{~g}, 0.01 \mathrm{~mol})$, in sodium ethoxide/ethanol solution [prepared by dissolving sodium metal (0.46 g, 0.02 $\mathrm{mol})$ in absolute ethanol $(60 \mathrm{~mL})$ ] was heated in a boiling water bath for $12 \mathrm{~h}$. The solid product formed upon pouring onto ice/water containing hydrochloric acid (till $\mathrm{pH} \mathrm{6)}$ was collected by filtration to give either $\mathbf{5 a}$ or $\mathbf{5 b}$, crystallized from DMF.

Method B. - To the dry solid of either 3a (3.09 g, $0.01 \mathrm{~mol})$ or $3 \mathrm{~b}(3.10 \mathrm{~g}, 0.01 \mathrm{~mol})$, aniline oil $(0.94 \mathrm{~g}, 0.01 \mathrm{~mol})$ was added. It was reaction mixture was heated in an oil bath $\left(140{ }^{\circ} \mathrm{C}\right)$ for $1 \mathrm{~h}$. It was left to $\mathrm{cool}$ and the remaining product was triturated with diethyl ether and the formed solid product was collected by filtration.

3-Benzoylmethylsulphido-1-imino-2-phenylaminocarbonyl-6,7,8,9-tetrahydrobenzo[b]-thieno[2,3-d]pyrimidine (6a), 3-benzoylmethylsulphido-1-oxo-2-phenylaminocarbonyl-6,7,8,9-tetrahydroben$z o[b]$ thieno[2,3-d]pyrimidine (6b), 1-imino-2-phenylaminocarbonyl-3-(ethyl acetatosulfyl)-6,7,8,9-tetrahydrobenzo[b]thieno[2,3-d]pyrimidine ( $7 a)$ and 1-oxo-2-phenylamino-carbonyl-3-(ethylacetatosulfyl)-6,7,8,9-tetrahydrobenzo[b]thieno[2,3-d]pyrimidine (7b)

General procedure. - To a solution of either $5 \mathrm{a}(3.56 \mathrm{~g}, 0.01 \mathrm{~mol})$ or $5 \mathbf{b}(3.57 \mathrm{~g}, 0.01$ $\mathrm{mol})$ in 1,4-dioxane $(40 \mathrm{~mL})$, either $\omega$-bromoacetophenone $(2.0 \mathrm{~g}, 0.01 \mathrm{~mol})$ or ethyl chloroacetate $(1.22 \mathrm{~g}, 0.01 \mathrm{~mol})$ was added. The reaction mixture, in each case, was heated under reflux for $2 \mathrm{~h}$, then poured onto ice/water containing a few drops of sodium hydroxide (to $\mathrm{pH}$ 6) and the formed solid product was collected by filtration to give either $\mathbf{6 a}, \mathbf{6 b}, 7 \mathbf{a}$ or $7 \mathbf{b}$, crystallized from 1,4-dioxane.

3-Hydrazino-2-phenylaminocarbonyl-1-imino-6,7,8,9-tetrahydrobenzo[b]thieno[2,3-d]pyrimidine (8a), 3-phenylhydrazino-2-phenylaminocarbonyl-1-imino-6,7,8,9-tetrahydrobenzo[b]thieno [2,3-d]pyrimidine (8b), 3-hydrazino-2-phenylaminocarbonyl-1-oxo-6,7,8,9-tetrahydrobenzo[b] thieno[2,3-d]pyrimidine (8c) and 3-phenylhydrazino-2-phenylaminocarbonyl-1-oxo-6,7,8,9-tetrahydrobenzo[b]thieno[2,3-d]pyrimidine $(8 d)$

General procedure. - To a solution of either $7 \mathbf{a}(4.42 \mathrm{~g}, 0.01 \mathrm{~mol})$ or $7 \mathbf{b}(4.43 \mathrm{~g}, 0.01$ $\mathrm{mol})$ in 1,4-dioxane $(40 \mathrm{~mL})$, either hydrazine hydrate $(0.5 \mathrm{~g}, 0.01 \mathrm{~mol})$ or phenylhydrazine $(1.08 \mathrm{~g}, 0.01 \mathrm{~mol})$ was added. The reaction mixture, in each case, was heated under reflux for $6 \mathrm{~h}$, then poured onto ice/water containing hydrochloric acid (to $\mathrm{pH}$ 6) and the formed solid product was collected by filtration to give either $\mathbf{8 a}$ or $\mathbf{8 b}$, crystallized from 1,4-dioxane or $8 \mathrm{c}$ or $\mathbf{8 d}$, crystallized from DMF. 
W. W. Wardakhan et al: Screening for antidepressant, sedative and analgesic activities of novel fused thiophene derivatives, Acta Pharm. 58 (2008) 1-14.

\section{Animals}

Swiss albino mice of either sex, weighing 20-25 g, aged 6-8 weeks, were supplied by the Animal House at National Research Centre (Giza, Egypt). Animals were maintained under 12/12 h light/dark cycle at $20 \pm 2$ and fed the standard laboratory diet and water ad libitum. In accordance with the recommendations for the proper care and use of laboratory animals (NIH publication No. 85-23, revised 1985), groups of 6 mice for group were used in all experiments.

\section{Screening for antidepressant activity}

Porsolt's forced-swimming test. - Each mouse was placed individually in a glass cylinder (diameter $12 \mathrm{~cm}$, height $24 \mathrm{~cm}$ ) filled with water to a height of $12 \mathrm{~cm}$. Water temperature was maintained at $22-23{ }^{\circ} \mathrm{C}$. The animal was forced to swim for $6 \mathrm{~min}$ and the duration of immobility was measured. The mouse was considered to be immobile when it stopped struggling and moved only to remain floating in water, keeping its head above water. The floating time, which was the measure of despair (21), was recorded 60 min after treatment with each drug (15 or $30 \mathrm{mg} \mathrm{kg}^{-1}$, i.p.), saline or imipramine $\left(15 \mathrm{mg} \mathrm{kg}^{-1}\right.$, i.p.). Test compounds were dissolved using a few drops of Tween 80 and further dilutions were done using saline to get the necessary doses. The negative control was the vehicle solution (Tween 80 in saline).

\section{Screening for sedative effect}

Mice were observed in a commercially available motor activity apparatus (Ugo Basel, Italy) in which locomotor and exploratory activity could be monitored. In these experiments, each mouse was intraperitoneally injected with the drug $\left(30 \mathrm{mg} \mathrm{kg}^{-1}\right)$ and $30 \mathrm{~min}$ later it was placed in the activity monitor in which activity was monitored for $6 \mathrm{~min}$.

\section{Screening for analgesic effect}

Acetic acid-induced writhing was performed for separate groups of 6 mice each, to which i.p. vehicle, compounds $\mathbf{2 a}, \mathbf{3 a}, \mathbf{5 b}, \mathbf{6} \mathbf{a}$ and $\mathbf{8 b}\left(15\right.$ and $\left.30 \mathrm{mg} \mathrm{kg}^{-1}\right)$ or indomethacin were administered $\left(20 \mathrm{mg} \mathrm{kg}^{-1}\right)$. After $30 \mathrm{~min}$ pretreatment interval, an i.p. injection of $0.6 \%$ acetic acid was administered (22). Each mouse was then placed in an individual clear plastic observational chamber and the total number of writhes (abdomen constriction, trunk twisting and extension of hind legs) made by each mouse was counted for 30 min after acetic acid administration.

\section{Statistics}

Data are presented as mean \pm SEM. Data were analyzed by ANOVA followed by the Duncan and multiple group comparison test. 
W. W. Wardakhan et al: Screening for antidepressant, sedative and analgesic activities of novel fused thiophene derivatives, Acta Pharm. 58 (2008) 1-14.

\section{RESULTS AND DISCUSSION}

\section{Chemistry}

The reaction of 2-amino-4,5,6,7-tetrahydrobenzo[b]thiophene derivative $\mathbf{1 a , b}$ with ethoxycarbonylisothiocyanate in 1,4-dioxane at room temperature gave the $N$-ethoxycarbonylthiourea derivatives $\mathbf{2} \mathbf{a}$ and $\mathbf{2} \mathbf{b}$, respectively. The structures of compounds $\mathbf{2} \mathbf{a}, \mathbf{b}$ were based on analytical and spectral data. Thus, the ${ }^{1} \mathrm{H}$ NMR spectrum of $2 \mathbf{a}$ showed the presence of a triplet at $\delta 1.61$ ppm corresponding to the ester $\mathrm{CH}_{3}$ group, a multiplet at $\delta$ 2.14-2.16 and 2.23-2.26 ppm corresponding to the four $\mathrm{CH}_{2}$ groups of cyclohexene

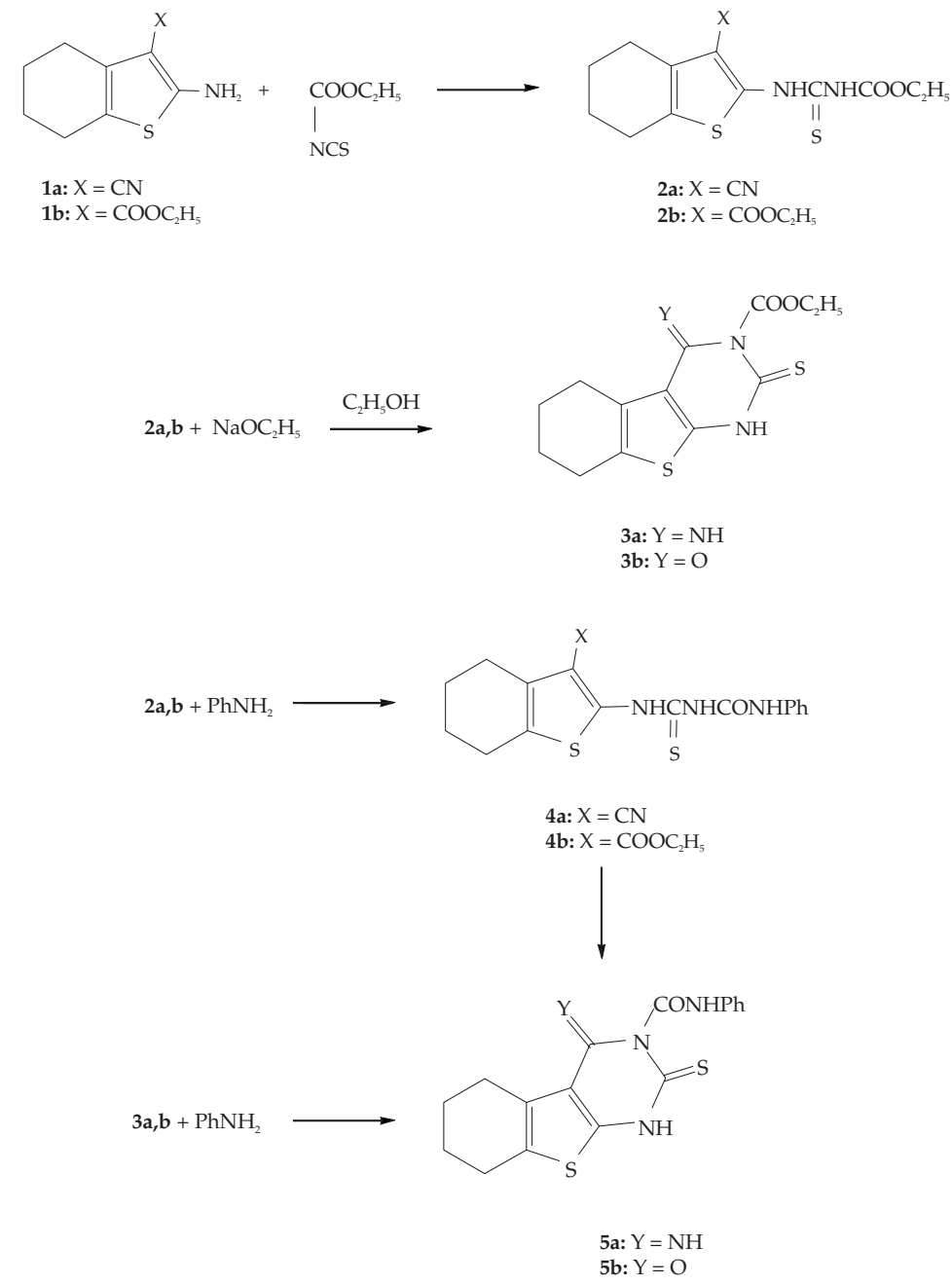

Sheme 1 
W. W. Wardakhan et al: Screening for antidepressant, sedative and analgesic activities of novel fused thiophene derivatives, Acta Pharm. 58 (2008) 1-14.

ring, two singlets at $\delta 4.11$ and 8.32 for the two $\mathrm{NH}$ groups and a quartet at $\delta 4.24 \mathrm{ppm}$ for the ester $\mathrm{CH}_{2}$ group. The reactions of isothiocyanates with $\mathrm{NH}_{2}$ compounds were reported earlier (23). Compounds $\mathbf{2} \mathbf{a}, \mathbf{b}$ underwent ready cyclization when heated in sodium ethoxide/ethanol solution in a boiling water bath to give the tetrahydro[b]thieno$[2,3-d]$ pyrimidine derivatives $3 a$ and $3 b$, respectively.

The reaction of $\mathbf{2} \mathbf{a}, \mathbf{b}$ with aniline in an oil bath (at $140^{\circ} \mathrm{C}$ ) gave the anilide derivatives $\mathbf{4} \mathbf{a}$ and $\mathbf{4 b}$, respectively. Structures of compounds $\mathbf{4 a} \mathbf{a} \mathbf{b}$ were based on the obtained analytical and spectral data (see experimental section). In the reaction of $\mathbf{2} \mathbf{b}$, aniline reacts with the ethyl $\mathrm{N}$-carboxylate rather than the ethyl carboxylate group attached to C-thiophene based on the cyclization of $4 \mathrm{~b}$ into $5 \mathrm{~b}$ via ethanol elimination. Moreover, formation of the same cyclized product via another reaction route was noted. Thus, compounds $\mathbf{4 a}$ and $\mathbf{4 b}$ underwent ready cyclization when heated in sodium ethoxide/ethanol solution in a boiling water bath to give the 4,5,6,7-tetrahydrobenzo[b]thieno[2,3-d]pyrimidine derivatives $\mathbf{5} \mathbf{a}$ and $\mathbf{5 b}$, respectively. Structures of the latter products were established on the basis of analytical and spectral data and on their synthesis using another reaction route. Thus, the reaction of either $3 \mathbf{a}$ or $3 \mathbf{b}$ with aniline oil at $140{ }^{\circ} \mathrm{C}$ gave the same products $\mathbf{5 a}$ and $\mathbf{5 b}$, respectively (m.p., mixed m.p. and fingerprint IR spectrum) (Scheme 1).

The reaction of either compound $\mathbf{5 a}$ or $\mathbf{5 b}$ with $\omega$-bromoacetophenone in 1,4-dioxane gave the thioether-tetrahydrobenzo[b]thieno[2,3-d]pyrimidine derivatives $\mathbf{6 a}$ and $\mathbf{6} \mathbf{b}$, respectively. Structures of the latter products were based on analytical and spectral data. The ${ }^{1} \mathrm{H}$ NMR spectrum of 6 a showed the presence of two multiplets with $\delta 1.65-1.70$ ppm and 2.22-2.31 ppm for the $4 \mathrm{CH}_{2}$ groups, a singlet with $\delta 4.68 \mathrm{ppm}$ for the side

Table III. Effect of tested compounds on the duration of immobility in Porsolt's forced-swimming test

\begin{tabular}{cc}
\hline Treatment/compd. & Duration of immobility $(\mathrm{s})^{\mathrm{a}}$ \\
\hline Saline & $289.9 \pm 7.1$ \\
Imipramine $\left(15 \mathrm{mg} \mathrm{kg}^{-1}\right)$ & $237.0 \pm 14.0^{\mathrm{b}}$ \\
2a $\left(15 \mathrm{mg} \mathrm{kg}^{-1}\right)$ & $279.7 \pm 11.4$ \\
2a $\left(30 \mathrm{mg} \mathrm{kg}^{-1}\right)$ & $265.5 \pm 15.8$ \\
3a $\left(15 \mathrm{mg} \mathrm{kg}^{-1}\right)$ & $269.3 \pm 9.6$ \\
3a $\left(30 \mathrm{mg} \mathrm{kg}^{-1}\right)$ & $266.1 \pm 10.3$ \\
$\mathbf{5 b}\left(15 \mathrm{mg} \mathrm{kg}^{-1}\right)$ & $271.7 \pm 8.5$ \\
$\mathbf{5 b}\left(30 \mathrm{mg} \mathrm{kg}^{-1}\right)$ & $266.7 \pm 17.6$ \\
$\mathbf{6 a}\left(15 \mathrm{mg} \mathrm{kg}^{-1}\right)$ & $286.8 \pm 7.8$ \\
$\mathbf{6 a}\left(30 \mathrm{mg} \mathrm{kg}^{-1}\right)$ & $273.3 \pm 13.6$ \\
$\mathbf{8 b}\left(15 \mathrm{mg} \mathrm{kg}^{-1}\right)$ & $283.9 \pm 11.8$ \\
$\mathbf{8 b}\left(30 \mathrm{mg} \mathrm{kg}^{-1}\right)$ & $301.0 \pm 9.4$ \\
\hline
\end{tabular}

a Mean \pm SEM $(n=6)$.

b Significant difference $v s$. saline-treated control group $(p<0.05)$. 
W. W. Wardakhan et al: Screening for antidepressant, sedative and analgesic activities of novel fused thiophene derivatives, Acta Pharm. 58 (2008) 1-14.

chain $\mathrm{CH}_{2}$ group, a multiplet with $\delta 7.33-7.39$ ppm for the two phenyl groups and two singlets with $\delta 8.38$ and 8.94 ppm corresponding to two NH groups. On the other hand, the reaction of either $\mathbf{5 a}$ or $\mathbf{5 b}$ with ethyl chloroacetate gave the thioether derivatives $\mathbf{7 a}$ and $\mathbf{7 b}$, respectively. The spectral data of the latter are consistent with the proposed structures (Tables I and II). The reaction of either compound $7 \mathbf{a}$ or $\mathbf{7 b}$ with either hydrazine hydrate or phenylhydrazine afforded the corresponding 2-hydrazino-4,5,6,7-tetrahydrobenzo[b]thieno[2,3-d]pyrimidine derivatives 8a-d, respectively (Scheme 2). The analytical and spectral data of the latter products agree well with the proposed structures (Tables I and II).

\section{Screening for antidepressant activity}

After 60 min of i.p. administration, some compounds (2a, 3a and $5 \mathbf{b}$ ) showed mild, non-significant antidepressant activity at high doses and were found active, compared with negative control group. The other compounds failed to display antidepressant properties in the swimming test (Table III).

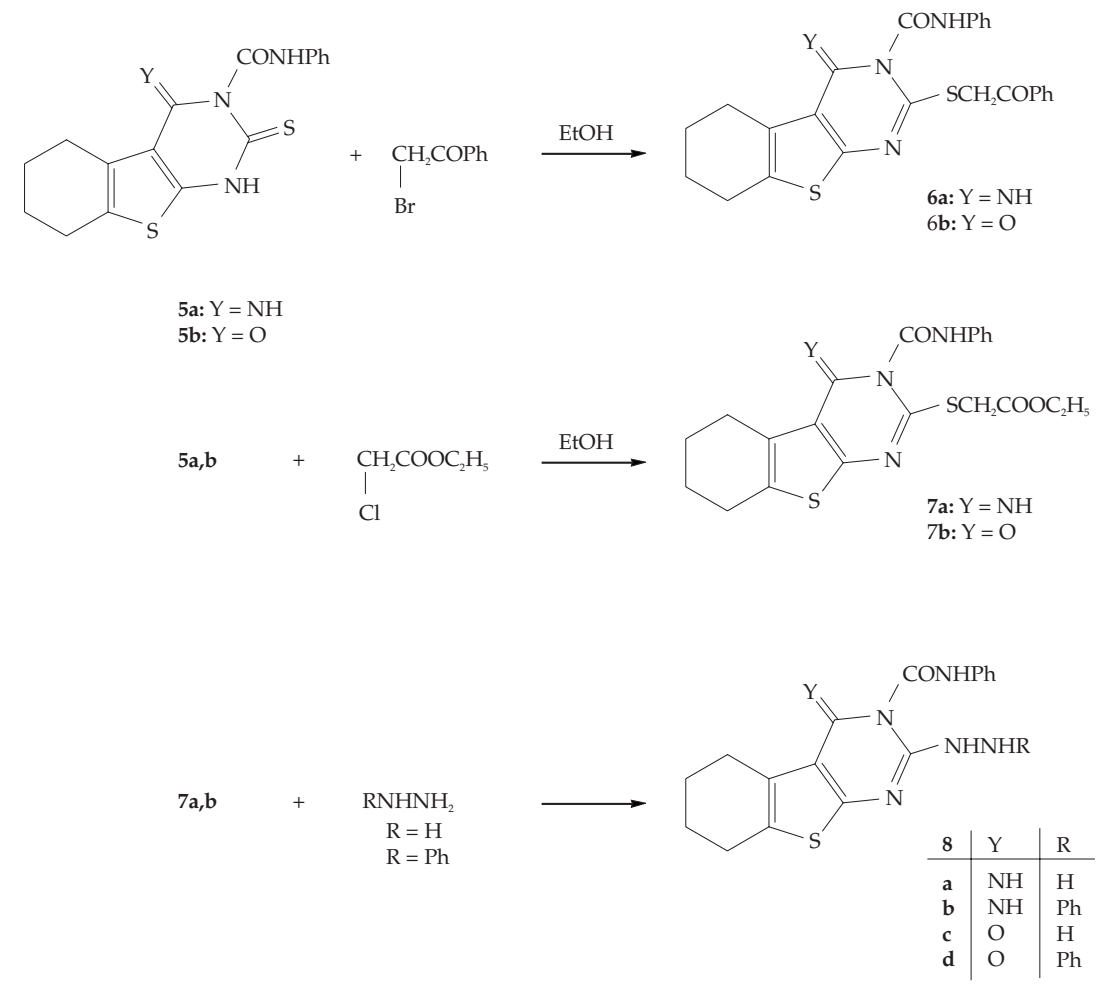

Sheme 2 
W. W. Wardakhan et al:: Screening for antidepressant, sedative and analgesic activities of novel fused thiophene derivatives, Acta Pharm. 58 (2008) 1-14.

\section{Screening for sedative activity}

No sedative effect could be observed in mice treated with compounds $\mathbf{2 a}, \mathbf{5 b}, \mathbf{6} \mathbf{a}$ and $\mathbf{8 b}$; even more exploratory movements compared to the saline treated group were observed (Table IV).

All tested compounds at the two doses (15 or $\left.30 \mathrm{mg} \mathrm{kg}^{-1}\right)$, except for the lower dose of $\mathbf{8 b}$, significantly reduced the number of abdominal writhes induced by i.p. injection of

Table IV. Effect of tested compounds on the number of exploratory movements in mice ${ }^{a}$

\begin{tabular}{cc}
\hline Treatment/compd. & Number of movements $^{\mathrm{b}}$ \\
\hline Saline & $27.8 \pm 2.3$ \\
2a $\left(30 \mathrm{mg} \mathrm{kg}^{-1}\right)$ & $36.3 \pm 3.9^{c}$ \\
3a $\left(30 \mathrm{mg} \mathrm{kg}^{-1}\right)$ & $25.0 \pm 1.9$ \\
5b $\left(30 \mathrm{mg} \mathrm{kg}^{-1}\right)$ & $51.3 \pm 3.0^{c}$ \\
6a $\left(30 \mathrm{mg} \mathrm{kg}^{-1}\right)$ & $39.3 \pm 4.4^{\mathrm{c}}$ \\
8b $\left(30 \mathrm{mg} \mathrm{kg}^{-1}\right)$ & $63.5 \pm 3.9^{c}$ \\
\hline
\end{tabular}

a Number of movements in 6 minutes.

b Mean \pm SEM $(n=6)$.

c Significant difference $v s$. saline-treated control group $(p<0.05)$.

Table V. Inhibition of abdominal constrictions caused by injection of acetic acid ${ }^{a}$

\begin{tabular}{|c|c|c|}
\hline Treatment/compd. & Number & Inhibition (\%) \\
\hline Saline & $92.8 \pm 6.0$ & - \\
\hline 2a $\left(15 \mathrm{mg} \mathrm{kg}^{-1}\right)$ & $44.8 \pm 4.0^{c}$ & 51.7 \\
\hline 2a $\left(30 \mathrm{mg} \mathrm{kg}^{-1}\right)$ & $2.5 \pm 0.29 c$ & 97.3 \\
\hline 3a $\left(15 \mathrm{mg} \mathrm{kg}^{-1}\right)$ & $28.3 \pm 3.5^{c}$ & 69.5 \\
\hline 3a $\left(30 \mathrm{mg} \mathrm{kg}^{-1}\right)$ & $26.8 \pm 1.2^{c}$ & 71.7 \\
\hline $5 \mathbf{b}\left(15 \mathrm{mg} \mathrm{kg}^{-1}\right)$ & $31.5 \pm 2.2^{c}$ & 66.1 \\
\hline $5 \mathbf{b}\left(30 \mathrm{mg} \mathrm{kg}^{-1}\right)$ & $27.5 \pm 3.2^{c}$ & 70.4 \\
\hline 6a $\left(15 \mathrm{mg} \mathrm{kg}^{-1}\right)$ & $50.0 \pm 4.0^{c}$ & 46.1 \\
\hline 6a $\left(30 \mathrm{mg} \mathrm{kg}^{-1}\right)$ & $23.5 \pm 2.6^{c}$ & 74.7 \\
\hline $8 \mathbf{b}\left(15 \mathrm{mg} \mathrm{kg}^{-1}\right)$ & $81.7 \pm 6.8$ & 11.9 \\
\hline $8 \mathbf{b}\left(30 \mathrm{mg} \mathrm{kg}^{-1}\right)$ & $57.3 \pm 4.1^{\mathrm{c}}$ & 38.3 \\
\hline Indomethacin $\left(20 \mathrm{mg} \mathrm{kg}^{-1}\right)$ & $50.3 \pm 5.4^{\mathrm{c}}$ & 45.8 \\
\hline
\end{tabular}

a Constriction during $30 \mathrm{~min}$.

$\mathrm{b}$ Mean $\pm \operatorname{SEM}(n=6)$.

c Significant difference vs. saline-treated control group $(p<0.05)$. 
W. W. Wardakhan et al:: Screening for antidepressant, sedative and analgesic activities of novel fused thiophene derivatives, Acta Pharm. 58 (2008) 1-14.

acetic acid in mice (Table V). Compound 2a was the most potent in this respect, inhibiting the number of abdominal writhes by $97.3 \%$ at high dose $\left(30 \mathrm{mg} \mathrm{kg}^{-1}\right)$, compared to saline as the negative control group. Meanwhile, compounds $\mathbf{6 a}, \mathbf{3} \mathbf{a}$ and $\mathbf{5 b}$ at high doses inhibited the number of abdominal writhes by $74.7,71.7$ and $70.4 \%$, respectively. These compounds, at low and high doses, were even more potent than indomethacin in this respect.

From the structure-activity relationship viewpoint, the thiourea tetrahydrobenzo[b]thiophene derivative $\mathbf{2 a}$ has the strongest antidepressant and analgesic activity. Replacement of ethoxycarbonylthiouryl side chain with fused pyrimidine moiety decreased these activities in the other compounds. The presence of benzoylmethylsulphide side chain attached to pyrimidine moiety in compound 6a increased its activity as analgesic agent.

\section{CONCLUSIONS}

In this study, we have described efficient synthesis of novel benzo[b]thienopyrimidine derivatives derived from the thiourea derivatives of tetrahydrobenzo[b]thiophenes $\mathbf{2 a}$ and $\mathbf{2} \mathbf{b}$. The newly synthesized thiophene derivatives $\mathbf{2 a}, \mathbf{3 a}, \mathbf{5 b}, \mathbf{6 a}$ and $\mathbf{8 b}$ showed mild antidepressant and analgesic activity of various intensities. However, compound $\mathbf{2 a}$ had the strongest antidepressant and analgesic activity. Compounds 2a, 6a, 3a and 5b, as analgesic agents, at low and high doses, were more potent than indomethacin.

\section{REFERENCES}

1. E. Palaska, M. Aytemir, I. T. Uzbay and D. Erol, Synthesis and antidepressant activities of some 3,5-diphenyl-2-pyrazolines, Eur. J. Med. Chem., 36 (2001) 539-543; DOI: 10.1016/S0223-5234(01) 01243-0.

2. M. F. Şahin, B. Badieogh, M. Gökçe, E. Küpeli, E. Yesilada, Synthesis and analgesic and antiinflammatory activity of methyl 6-substituted-3(2H)-pyridazinone-2-yl acetate derivatives, Arch. Pharm. 337 (2004) 445-452.

3. A. A. Bilgin, E. Palaska, R. Sunal and B. Gumusel, 1,3,5-Triphenyl-2-pyrazolines with antidepressant activities, Pharmazie 49 (1994) 67-69.

4. O. M. E. Abdel-Salam and S. M. Nofal and S. M. El-Shenawy, Evaluation of the anti-inflammatory and anti-nociceptive effects of different antidepressants in the rats, Pharmacol. Res. 48 (2003) 157-165; DOI: 10.1016/S1043-6618(03)00106-3.

5. M. Vrethem, J. Boivie, H. Arnqvist, H. Holmgren, T. Lindstrom and L. H. Thorell, A comparison of amitriptyline and maprotiline in the treatment of painful polyneuropathy in diabetics and non-diabetics, Clin. J. Pain 13 (1997) 313-323; DOI: 10.1079/00002508-199712000-00009.

6. S. S. Bahekar and D. B. Shinde, Synthesis and anti-inflammatory activity of some [4,6-(4-substiuted aryl)-2-thioxo-1,2,3,4-tetrahydro-pyrimidin-5-yl]-acetic acid derivatives, Bioorg. Med. Chem. Lett. 14 (2004) 1733-1736.

7. J. P. Beck, M. A. Curry, R. J. Chorvat, L. W. Fitzgerald, P. J. Gilligan, R. Zaczek and G. L. Trainor, Thiazolo[4,5-d]pyrimidine thiones and -ones as corticotropin-releasing hormone (CRH-R1) receptor antagonists, Bioorg. Med. Chem. Lett. 9 (1999) 1185-1188. 
8. V. Alagarsamy, S. Meena, K. V. Ramseshu, V. R. Solomon, K. Thirumurugan, K. Dhanabal and M. Murugan, Synthesis, analgesic, anti-inflammatory, ulcerogenic index and antibacterial activities of novel 2-methylthio-3-substituted-5,6,7,8-tetrahydrobenzo-(b)thieno[2,3-d]pyrimidin-4 (3H)-ones, Eur. J. Med. Chem. 41 (2006) 1293-1300.

9. W. W. Wardakhan, H. M. Gaber, S. A. Ouf and S. M. Sherif, Studies on 2-aminothiophenes: synthesis, transformations and biological evaluation of functionally substituted thiophenes and their fused derivatives, Phosph. Sul. Sil. 180 (2005) 601-618; DOI: 10.1080/104265090517406.

10. W. W. Wardakhan, H. Z. Shams and H. E. Moustafa, Synthesis of polyfunctionally substituted thiophene, thieno[2,3-b]pyridine and thieno[2,3-d]pyrimidine derivatives, Phosph. Sul. Sil. 180 (2005) 1815-1827; DOI: 10.1080/104265090889422.

11. W. W. Wardakhan, G. A. Elmegeed, F. M. Manhi, Utility of 2-aminothiophene-3-carboxamide in the synthesis of biologically active fused heterocyclic derivatives, Phosph. Sul. Sil. 180 (2005) 125-140; DOI: 10.1080/104265090889422.

12. W. W. Wardakhan and S. A. Ouf, Reaction of cyanoacetamide with benzoyl isothiocyanate: synthesis of pyridine, pyrido[2,2-a]-1,2,3-triazine, pyrazolo[3,4-d]-1,2,3-triazine and thiazole derivatives, Egypt. J. Chem. 48 (2005) 393-406.

13. J. Graff, S. Harder, O. Wahl, E. H. Scheuermann and J. Gossmann, Anti-inflammatory effects of clopidogrel intake in renal transplant patients: effects on platelet-leukocyte interactions, platelet CD40 ligand expression, and proinflammatory biomarkers, Clin. Pharmacol. Ther. 78 (2005) 468-476.

14. A. Hymete, J. Rohloff, H. Kjosen and T. H. Iversen, Acetylenic thiophenes from the roots of Escinops ellenbeckii from Ethiopia, Nat. Prod. Res. 19 (2005) 755-761; DOI: 10.1080/ 1478641042000301711.

15. R. A. Tapia, L. Alegria, C. D. Pessoa, C. Salas, M. J. Cortes, J. A. Valderrama, M. E. Sarciron, F. Pautet, N. Walchshofer and H. Fillion, Synthesis and antiprotozoal activity of naphthofuranquinones and naphthothiophenequinones containing a fused thiazole ring, Bioorg. Med. Chem. 11 (2003) 2175-2182; DOI: 10.1016/S0968-0896(03)00122-6.

16. P. Dallemagne, L. P. Khanh, A. Alsaidi, I. Varlet, V. Collot, M. Paillet, R. Bureau and S. Rault, Synthesis and biological evaluation of five-membered heterocycles fused to cyclopenta[c]thiophene as new antitumer agents, Bioorg. Chem. 11 (2003) 1161-1167; DOI: 10.1016/S0968-0896(02) 00654-5.

17. M. E. Migaud, R. C. Wilmouth, G. I. Mills, G. J. Wayne, C. Risley, C. Chambers, S. J. F. Macdonald and C. J. Schofield, 5,5-Fused thiophene gamma-lactams as templates for serine protease inhibition, Chem. Com. 12 (2002) 1274-1275.

18. M. Pietsch and M. Gutschow, Alternate substrate inhibition of cholesterol esterase by thieno [2,3-d][1,3]oxazin-4-ones, J. Biol. Chem. 277 (2002) 24006-24013.

19. R. M. Mohareb and F. M. Manhi, Reaction of 2-diazo-4,5,6,7-tetrahydro-benzo[b]thiophene-3carboxylate with 3-iminobutyronitrile: synthesis of thiophene and their fused derivatives, Heteroatom. Chem. 11 (2000) 403-412; DOI: 10.1002/1098-1071(2000).

20. W. W. Wardakhan, N. A. Louca and M. M. Kamel, The reaction of 2-aminocyclohexeno-[b]thiophene derivatives with ethoxycarbonylisothiocyanate: synthesis of fused thiophene derivatives with antimicrobial and antifungal activities, Acta Chim. 54 (2007) 229-241.

21. R. D. Porsolt, M. Le Pichon and M. Jalfre, Depression: a new animal model sensitive to antidepressant treatments, Nature 266 (1977) 730-732; DOI: 10.1038/266730a0.

22. R. Koster, M. Anderson and E. J. De Beer, Acetic acid for analgesic screening, Fed. Proc. 18 (1959) 412-413.

23. S. Reyes and K. Burgess, On formation of thiohydantoins from amino acids under acylation conditions, J. Org. Chem. 71 (2006) 2507-2509; DOI: 10.102/jo052576. 
W. W. Wardakhan et al.: Screening for antidepressant, sedative and analgesic activities of novel fused thiophene derivatives, Acta Pharm. 58 (2008) 1-14.

\section{$S A \check{Z} E T A K$}

\section{Ispitivanje antidepresivnog, sedativnog $\mathrm{i}$ analgetskog djelovanja novih fuzioniranih derivata tiofena}

WAGNAT W. WARDAKHAN, OMAR M. E. ABDEL-SALAM i GAMAL A. ELMEGEED

U radu je opisana sinteza fuzioniranih derivata benzotiofena koji sadrže heterociklički ostatak bitan za farmakološko djelovanje. Tiourea derivati 2a,b dobiveni su reakcijom derivata tetrahidrobenzo[b]tiofena $\mathbf{1 a , b}$ s etoksikarbonilizotiocijanatom. Iz njih su dalje priređeni anulirani derivati benzo[b]tienopirimidina $\mathbf{3 a}, \mathbf{b}$. Spojevi $\mathbf{2} \mathbf{a}, \mathbf{b}$ i $\mathbf{3 a}$ su reakcijama heterociklizacije prevedeni $u$ benzo[b]tienopirimidine $\mathbf{5 a}, \mathbf{b}-\mathbf{8} \mathbf{a}-\mathbf{c}$. Ispitivano je antidepresivno, sedativno i analgetsko djelovanje novosintetiziranih derivata tiouree $\mathbf{2 a}$ i benzo[b]tienopirimidina 3a, 5b, 6a i $\mathbf{8 b}$ u dvije doze (15 ili $30 \mathrm{mg} \mathrm{kg}^{-1}$ tjelesne mase). Spojevi 2a, 3a i 5b pokazali su blago antidepresivno djelovanje u testu forsiranog plivanja, dok sedativni učinak nije pokazao niti jedan ispitivani spoj. Visceralna bol inducirana i.p. injekcijom octene kiseline u miševa značajno je inhibirana sa svim spojevima, ali $u$ visokim dozama.

Ključne riječi: tetrahidrobenzo[b]tiofen, pirimidin, tiourea, antidepresiv, sedativ, analgetik

National Organization for Drug Control and Research, P.O. 29, Cairo, Egypt

Pharmacology Department, Hormone Department, National Research Centre, Dokki, Cairo, Egypt 